\title{
Description and Flow Assessment of the Delft Hypersonic Ludwieg Tube
}

\author{
Ferry F.J. Schrijer* \\ Willem J. Bannink ${ }^{\dagger}$ \\ Delft University of Technology, Kluyverweg 1,2629HS, Delft, The Netherlands
}

\begin{abstract}
An assessment of the hypersonic Ludwieg Tube of Delft University of Technology (Hypersonic Test Facility Delft, HTFD) is given. The facility is discussed theoretically and an experimental evaluation is performed to infer the facility performance. Experiments are performed using conventional techniques such as static and total head pressure measurements and Fay-Riddell heat flux evaluations by means of infrared thermography. Furthermore PIV (particle image velocimetry), a more state of the art technique is used to deduce nozzle boundary layer parameters as well as the free stream flow field and the static and total temperature for the Mach 7 nozzle. Finally for the Mach 9 nozzle, stagnation heat flux measurements were performed to obtain the total temperature of the flow.
\end{abstract}

\section{Introduction}

This paper discusses the hypersonic test facility Delft (HTFD), which is a Ludwieg expansion tube with a circular cross section. The Ludwieg tube tunnel concept was first conceived by H. Ludwieg ${ }^{1}$ in 1955 . Originally Ludwieg designed the facility as a low cost alternative for subsonic/transonic testing at high Reynolds numbers. The advantage of a Ludwieg tube facility is the ability to create a low turbulence uniform free stream. Later it was used for hypersonic applications. ${ }^{2,3}$ The appeal of this kind of facility to the hypersonic flow regime lies in the relatively long run times $(0.1$ to 0.2 seconds), large test section $(30 \mathrm{~cm})$ and high Reynolds numbers (order of $5-50 \times 10^{6} \mathrm{~m}^{-1}$ ).

The Ludwieg tube consists of four basic sections: storage tube, nozzle, test section and vacuum tank. The high pressure, high temperature storage tube is separated from the downstream part of the tube consisting of a nozzle and test section by a fast-opening valve, see figure 1. When the valve opens, air flows from the storage tube through the nozzle into the test section. The expansion ratio of the nozzle determines the Mach number in the test section.

The measurement of free stream variables has always shown to be challenging in short duration facilities. For the determination of the Reynolds number and free stream enthalpy an accurate determination of the free stream variables is needed. These can be obtained directly by measuring the static flow quantities. As an example the static pressure is normally obtained by measuring it on a flat plate or cone, however the value is extremely sensitive for any model misalignment with respect to the free stream. As an alternative the total values can be used which then also raises the need for an accurate determination of the Mach number. The static or total temperature is difficult to measure in short duration facilities due to the thermal inertia of the probes that are used.

In this paper a theoretical description is given on the operating principle of the Ludwieg tube. Theoretical estimates are given for the run time, total temperature and total free stream pressure. Furthermore measurements are performed to assess the performance of the facility. Among the measurements are the determination of the wind tunnel nozzle boundary layer just upstream of the test section and the total wind tunnel run time. An accurate determination of the free stream characteristics (total temperature, total pressure and Mach number) is made for a specified calibration point and the total operating Mach-Reynolds

*Assistant Professor, Faculty of Aerospace Engineering, Delft.

${ }^{\dagger}$ Associate Professor (Emeritus), Faculty of Aerospace Engineering, Delft. 


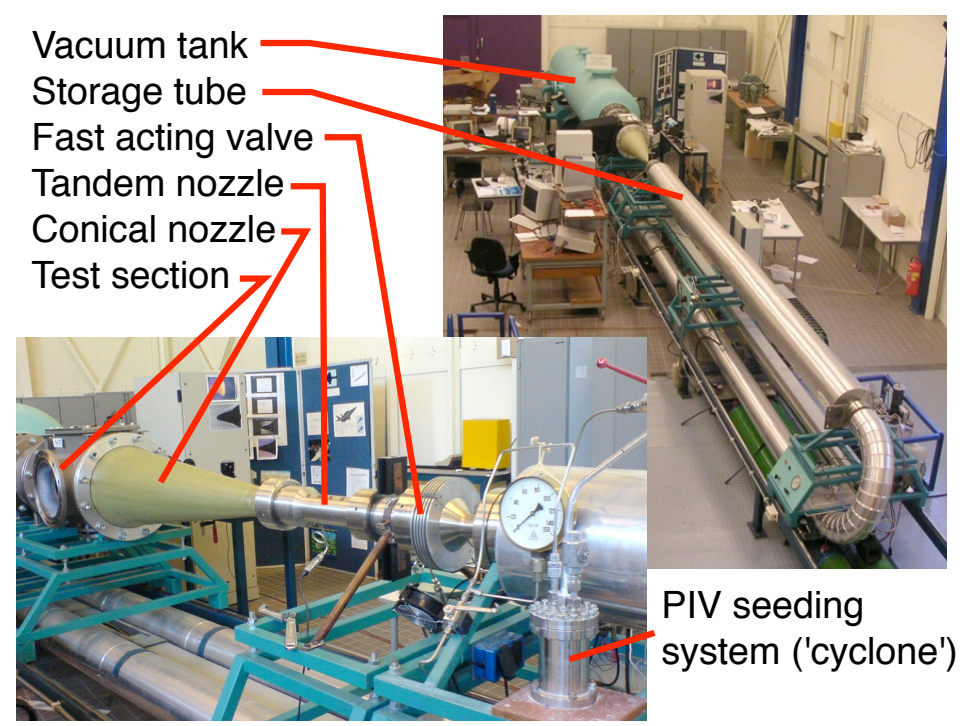

Figure 1. Overview of the HTFD

envelope is given. Finally the effect and limits of free stream condensation is addressed which is pertinent to these types of facility.

\section{Operating principles of the Ludwieg Tube}

The air used in the experiments is stored in the storage tube under high pressure and high temperature. As a result of the opening of the valve an expansion wave travels into the storage tube. The flow conditions behind the expansion wave act as storage tube conditions for the flow in the tunnel. Since the valve opening is relatively quick ${ }^{4}$ the expansion wave can be described by "simple wave" theory. ${ }^{5}$ The flow velocity $u_{1}$ in the tube can be obtained from:

$$
\frac{u_{1}}{a_{0}}=\frac{M_{1}}{1+\frac{\gamma-1}{2} M_{1}}
$$

where $u_{1}+\frac{2 a_{1}}{\gamma-1}=\frac{2 a_{0}}{\gamma-1}$ along a downstream characteristic, see figure 2, $a_{0}$ is the speed of sound based on storage tube conditions and $M_{1}$ is the resulting Mach number in the storage tube.

Region 0 corresponds to the flow conditions in front of and region 1 corresponds to conditions behind the expansion wave. The total temperature ratio over the expansion wave may be derived as:

$$
\frac{T_{t, 1}}{T_{0}}=\frac{1+\frac{\gamma-1}{2} M_{1}^{2}}{\left(1+\frac{\gamma-1}{2} M_{1}\right)^{2}}
$$

corresponding to a total pressure ratio by:

$$
\frac{p_{t, 1}}{p_{0}}=\left\{\frac{1+\frac{\gamma-1}{2} M_{1}^{2}}{\left(1+\frac{\gamma-1}{2} M_{1}\right)^{2}}\right\}^{\frac{\gamma}{\gamma-1}}
$$

The total running time of the facility with constant storage tube conditions may be calculated as:

$$
t_{1}=\frac{L}{a_{0}} \frac{2}{1+M_{1}}\left(1+\frac{\gamma-1}{2} M_{1}\right)^{\frac{\gamma+1}{2(\gamma-1)}}
$$

Finally the Mach number of the flow downstream of the expansion wave is determined by conservation of mass:

$$
\left(\frac{d_{\text {tube }}}{d^{*}}\right)^{2}=\frac{1}{M_{1}}\left[\frac{2}{\gamma+1}\left(1+\frac{\gamma-1}{2} M_{1}^{2}\right)\right]^{\frac{\gamma+1}{2(\gamma-1)}}
$$




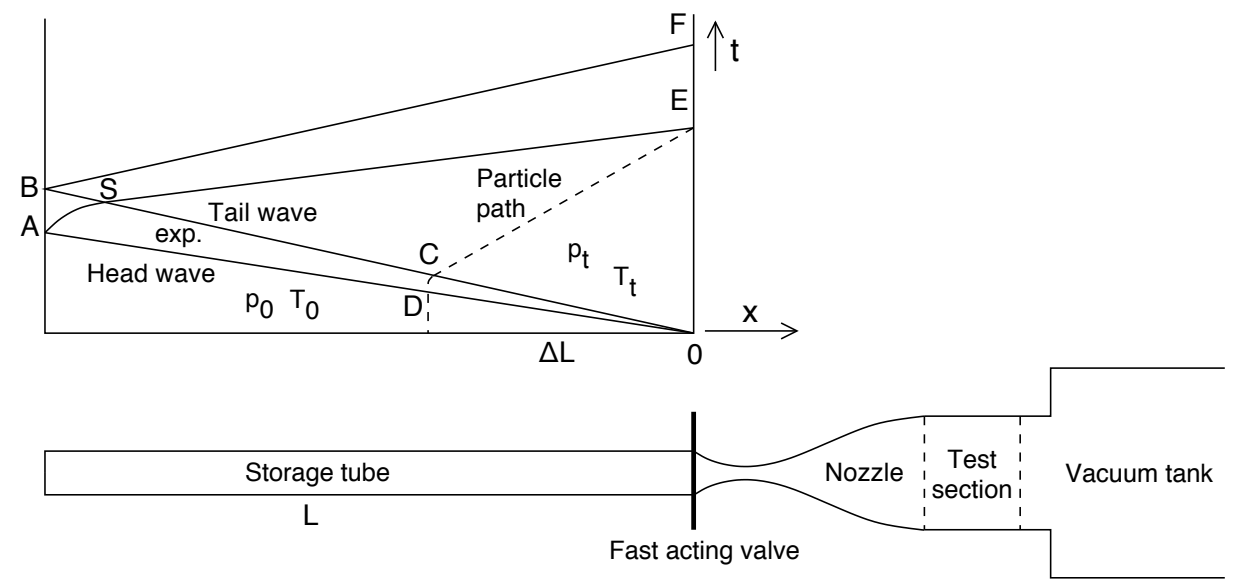

Figure 2. Operational principle of the Ludwieg tube

where the subscript $*$ denotes the critical (sonic) diameter of the throat section. The effects of free stream Mach number on the variation of total quantities and run time is given in table 1. For free stream Mach numbers less than 9 a tandem nozzle must be used, see section III, where the first section consists of the Mach 9 nozzle. Therefore only the results for Mach numbers 9 and higher are listed. The table shows an increase in losses of total flow quantities and runtime with decreasing Mach number where the Mach 9 case is the most unfavourable. However, it is found that the total temperature loss at the fast-opening valve dominates (see section IV.A).

\begin{tabular}{|c|cccc|}
\hline$M$ & $M_{1}$ & $\frac{T_{t, 1}}{T_{0}}$ & $\frac{p_{t, 1}}{p_{0}}$ & $\frac{t_{1} a_{0}}{L}$ \\
\hline 9 & 0.09 & 0.97 & 0.89 & 1.94 \\
10 & 0.05 & 0.98 & 0.93 & 1.96 \\
11 & 0.03 & 0.99 & 0.95 & 1.97 \\
\hline
\end{tabular}

Table 1. Expansion wave characteristics

To prevent condensation in the free stream, the air is heated in the storage tube. In order to increase the run time of the tunnel and to reduce the amount of energy required only a section of the storage tube is heated that contains the air that is used during the run. The furthest upstream location of a particle that can reach the valve within the run-time (path DCE in figure 2) can be approximated by:

$$
\Delta L=M_{1} \frac{a_{1}-u_{1}}{a_{0}} 2 L
$$

For a total tube length of $L=29 \mathrm{~m}$ (see table 2) a distance of $\Delta L=5.2 \mathrm{~m}$ results. Therefore only the first $6 \mathrm{~m}$ of the hot tube is heated.

The temperature in the storage tube has a maximum of $773 \mathrm{~K}\left(500^{\circ} \mathrm{C}\right)$ due to legislation issues. For high temperatures the specific heat ratio $\gamma$ is no longer constant since the vibrational degree of freedom becomes important. For $T=773 \mathrm{~K}$ the value for $\gamma=1.36^{6}$ which shows that variations in $\gamma$ are not important in considering the flow process.

The discontinuity between the high and low temperature part may cause a reflection of the expansion wave unless special precautions are taken. The reflection can be mitigated by changing the tube cross section 


\begin{tabular}{|l|r|}
\hline Total length storage tube & $L_{\text {tot }}=29 \mathrm{~m}$ \\
Length hot tube & $L_{\text {hot }}=6 \mathrm{~m}$ \\
Length cold tube & $L_{\text {cold }}=23 \mathrm{~m}$ \\
Diameter test section & $D=350 \mathrm{~mm}$ \\
Diameter hot tube & $D_{\text {hot }}=49.25 \mathrm{~mm}$ \\
Diameter cold tube & $D_{\text {cold }}=59 \mathrm{~mm}$ \\
\hline
\end{tabular}

Table 2. HTFD dimensions

accordingly effectively keeping the mass flow constant. Since the cross sections of the hot and cold part are given (see table 2), the temperature should be set according to: ${ }^{7}$

$$
\frac{d_{\text {hot }}}{d_{\text {cold }}}=\left(\frac{T_{\text {cold }}}{T_{\text {hot }}}\right)^{\frac{1}{4}}
$$

Hence for a hot tube temperature of $T_{\text {hot }}=773 \mathrm{~K}$, a cold tube temperature of $T_{\text {cold }}=375 \mathrm{~K}$ is needed.

In figure 3 a typical pressure variation in time in the storage tube is given. The pressure transducer is located just upstream of the valve and measures the static pressure. Initially the pressure is equal to the storage tube pressure which in this case is $p_{0}=99.8 \mathrm{bar}$, subsequently the pressure drops due to the expansion wave to approximately $83 \mathrm{bar}$. Since the sensor is flush mounted with the tube wall the static pressure $p_{1}$ is measured, however since the Mach number in the tube is low (see table 1) this is practically equal to the total pressure behind the expansion wave $p_{t, 1}$. The rms pressure fluctuation in the storage tube after the expansion wave has passed is 0.3 bar.

After approximately $25 \mathrm{~ms}$ a small oscillation is detected, which is caused by a partial reflection coming from the temperature/cross section discontinuity. Progressing further in time, a slow pressure rise is observed which is attributed to the cold air that is heated under constant volume when it enters the hot tube.

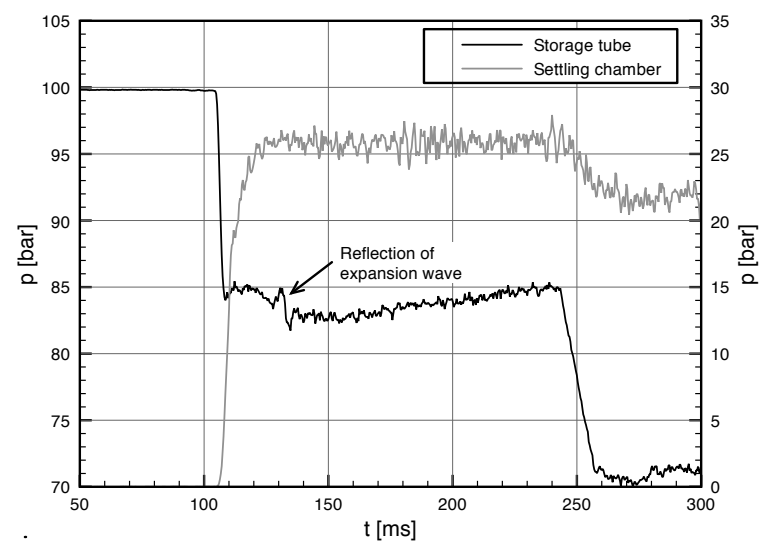

Figure 3. Pressure variation in time in the hot part of the storage tube and in the settling chamber

\section{Nozzle flow}

The nozzle has a conical shape with a $15^{\circ}$ total opening angle. The free stream Mach number is varied by changing the throat section. Various throat sections are available, see table 3. For the Mach 10 and 11 nozzles free stream condensation could become a problem, however since the parts are available for the facility they are included in the discussion.

\begin{tabular}{|c|cccccc|}
\hline$M$ & 6 & 7 & 8 & 9 & 10 & 11 \\
\hline$d^{*}$ & 48.0 & 34.3 & 25.38 & 19.35 & 15.12 & 12.06 \\
\hline
\end{tabular}

Table 3. Throat diameters in $\mathrm{mm}$ for the HTFD 


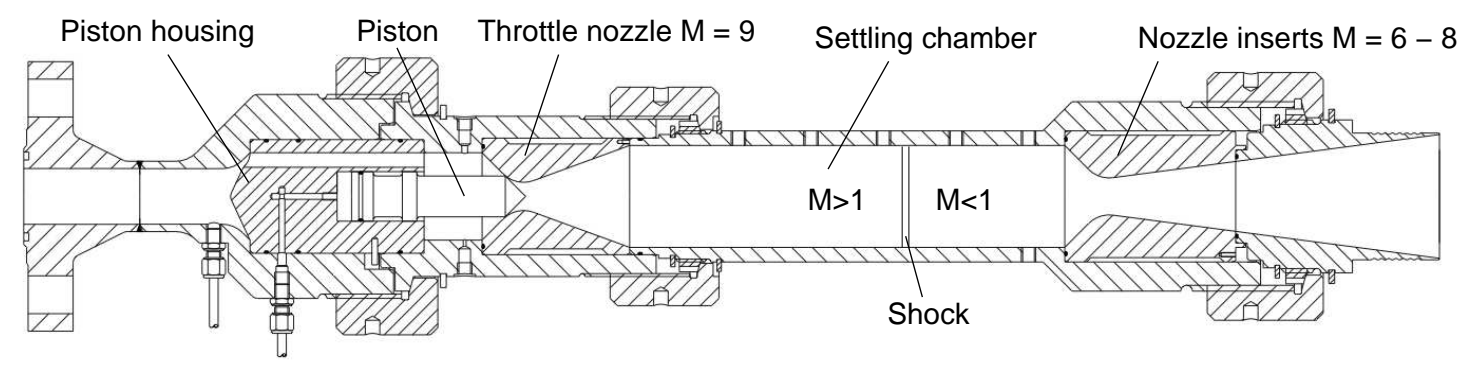

Figure 4. HTFD tandem nozzle block

For Mach numbers larger than 9 the throat section directly connects to the conical nozzle, while for smaller Mach numbers, an extra throttle nozzle is needed. This is because the throat diameter is limited by the smallest cross section present in the valve. If the throat cross section is larger, sonic conditions in the throat cannot be reached. The first part of the system is a Mach 9 nozzle which accelerates the flow to supersonic speeds after which it is decelerated to subsonic speeds through a number of shocks. The second throat reaccelerates the flow to the desired Mach number, see figure 4. Due to the shock waves in the tandem nozzle a total pressure loss occurs, which can be quantified by considering the total pressure loss over a normal shock wave:

$$
\frac{p_{t, 2}}{p_{t, 1}}=\left(\frac{d_{1}^{*}}{d_{2}^{*}}\right)^{2}
$$

The equation results for mass conservation in an adiabatic flow. In practice $p_{t, 1}$ is the total pressure in the storage tube and $p_{t, 2}$ equals the free stream total pressure. The pressure in the settling chamber between the two throats for a free stream Mach number of 7 is measured and it is indicated in figure 3 . The measured local static pressure is $25 \pm 1$ bar. The total pressure is obtained using the local Mach number that is calculated from the ratio of the local cross section (diameter is $70 \mathrm{~mm}$ ) with respect to the throat cross section. The local Mach number is 0.44 , this in combination with the measured static pressure results in a total pressure of $p_{t, 2}=28.8 \pm 1 \mathrm{bar}$. The measured pressure ratio now becomes $p_{t, 2} / p_{t, 1}=28.8 / 83.0=0.347$. The geometrical area ratio is $\left(d_{1}^{*} / d_{2}^{*}\right)^{2}=(19.35 / 34.3)^{2}=0.318$, this is slightly lower than the theoretical value. Apparently there is a shock train in the tandem nozzle block that causes less total pressure loss compared to a normal shock.

\section{III.A. Nozzle boundary layer}

Downstream of the throat the nozzle length is determined by the throat-to-test-section area ratio and total opening angle $\left(15^{\circ}\right)$, for Mach 7 this is $1.18 \mathrm{~m}$. Theoretically the area ratio determines the free stream Mach number. However in hypersonic facilities the displacement effect of the nozzle boundary layer can decrease the area ratio causing a lower free stream Mach number. To infer the boundary layer thickness at the end of the nozzle, PIV measurements ${ }^{8,9}$ were performed for the Mach 7 nozzle. The measurement location is indicated in figure 5. Since the optical access to the nozzle was limited, only a single camera could be used. Additionally the viewing angle with respect to the illuminated plane was large $\left(>45^{\circ}\right)$ in which case an out-of-plane velocity component may introduce a perspective error. ${ }^{10}$ Although the absolute magnitude of the velocity is less accurate, the relative values still enable to extract a boundary velocity profile to assess the integral boundary layer quantities. The extracted profile is shown in figure 6 and a 7 th order polynomial line is fitted. The compressible boundary layer integral quantities were obtained using the adiabatic Crocco-Busemann relation relating the velocity profile to the density distribution. ${ }^{11}$

The boundary layer parameters are given in table 4 . Due to the effect of boundary layer displacement the area ratio is decreased from 104.1 to 85.8 resulting in a Mach number decrease from 7 to 6.7. This also affects the maximum divergence encountered in the free stream, using the boundary layer correction this decreases from $7.5^{\circ}$ to $7.0^{\circ}$. The boundary layer corrected quantities will also be used in the remainder of the discussion. 


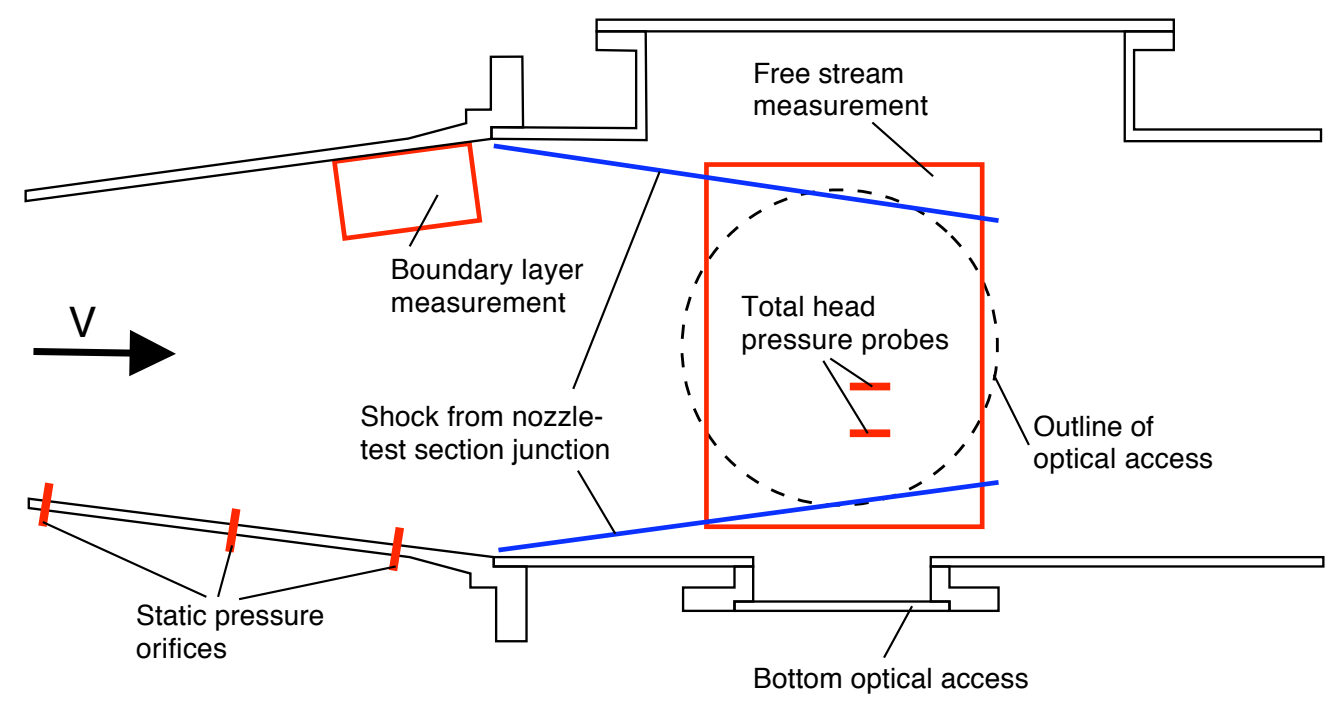

Figure 5. Overview of the nozzle and test section including the measurement locations

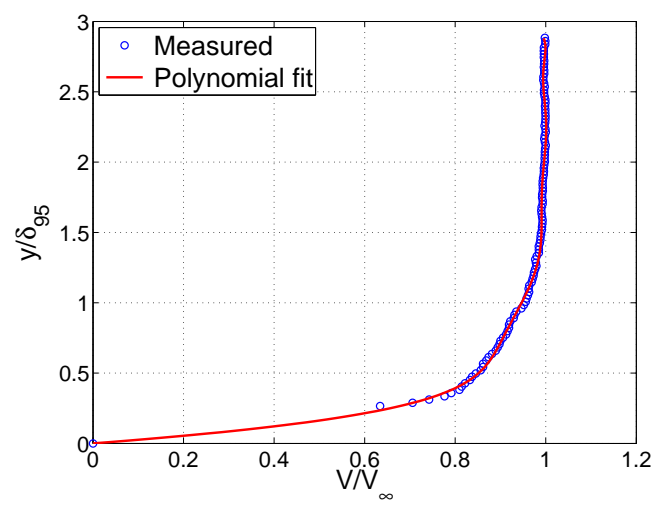

Figure 6. Boundary layer profile at the end of the conical Mach 7 nozzle

\section{Free stream assessment}

When the flow enters the test section it keeps diverging due to the conical nozzle. Therefore, the Mach number in the test section will be higher than obtained from the theoretical area relation. For the Mach 7 nozzle the theoretical Mach number in the test section is $M_{\infty}$,theory $=7.7$ after correcting for the boundary layer displacement thickness this is $M_{\infty, \text { cor }}=7.5$. The local Mach number variation due to the conical flow field in the center of the test section may be calculated by:

$$
\frac{\mathrm{d} M}{\mathrm{~d} x}=\frac{2 M\left(1+\frac{\gamma-1}{2} M^{2}\right)}{M^{2}-1} \frac{\tan \phi}{r}
$$

Where $\phi$ is the nozzle half angle and $r$ is the test section radius. For $M=7.5$ and $\phi=7^{\circ}, \frac{\mathrm{d} M}{\mathrm{~d} x}=0.023 \mathrm{~cm}^{-1}$. The test section radius is obtained from the Mach area-ratio relation:

$$
\frac{A}{A^{*}}=\frac{1}{M}\left(\frac{1+\frac{\gamma-1}{2} M^{2}}{\frac{\gamma+1}{2}}\right)^{\frac{\gamma+1}{2(\gamma-1)}}
$$

For a typical model having a length of $L=10 \mathrm{~cm}$ a $\Delta M=0.23$ results.

Since the test section has a cylindrical shape, a shock wave is formed at the nozzle test-section junction (figure 5). For $M=6.7$ (Mach number at the location of the nozzle test-section junction) and a compression angle of $\phi=7^{\circ}$ a shock with an angle of $\theta=14^{\circ}$ with respect to the free stream is formed, the shock angle with respect to the test section wall is $7^{\circ}$. 


\begin{tabular}{|lrl}
\hline Geometrical cross section & $\left(\frac{A}{A^{*}}\right)_{\text {geo }}$ & $=104.1$ \\
Theoretical Mach number & $M_{\text {theory }}$ & $=7$ \\
Corrected cross section & $\left(\frac{A}{A^{*}}\right)_{\text {cor }}$ & $=85.8$ \\
Corrected Mach number & $M_{\text {cor }}$ & $=6.7$ \\
Unit Reynolds number & $R e / m$ & $=16.2 \times 10^{6} \mathrm{~m}^{-1}$ \\
$95 \%$ boundary layer thickness & $\delta_{95}$ & $=13 \mathrm{~mm}$ \\
Displacement thickness & $\delta^{*}=11 \mathrm{~mm}$ \\
Momentum loss thickness & $\theta=0.63 \mathrm{~mm}$ \\
Shape parameter & $H=18$ \\
\hline
\end{tabular}

Table 4. Boundary layer parameters for the Mach 7 nozzle flow

In figure 7 an overview of the vertical component of the free stream flow field in the test section is shown. In order to visualize the junction shock the camera was oriented under a large angle with respect to the illuminated plane requiring a stereo PIV setup. The free stream part of the flow was measured using PIV at the location shown in figure 5 .

The black circle in figure 7 shows the outline of the windows. By plotting the vertical velocity component the junction shocks are clearly visualized. The shock at the bottom has an angle of $9^{\circ}$ while the angle at the top is $10^{\circ}$. The difference in shock angle between the upper and lower part of the test section is the presence of a considerably larger cavity at the top accommodating for the model support system. It is conceived that the large cavity has a considerable effect on the flow causing a larger shock angle. However it has no influence on the free stream flow, in the center of the test section a region of $200 \times 200 \mathrm{~mm}^{2}$ of undisturbed flow is guaranteed.
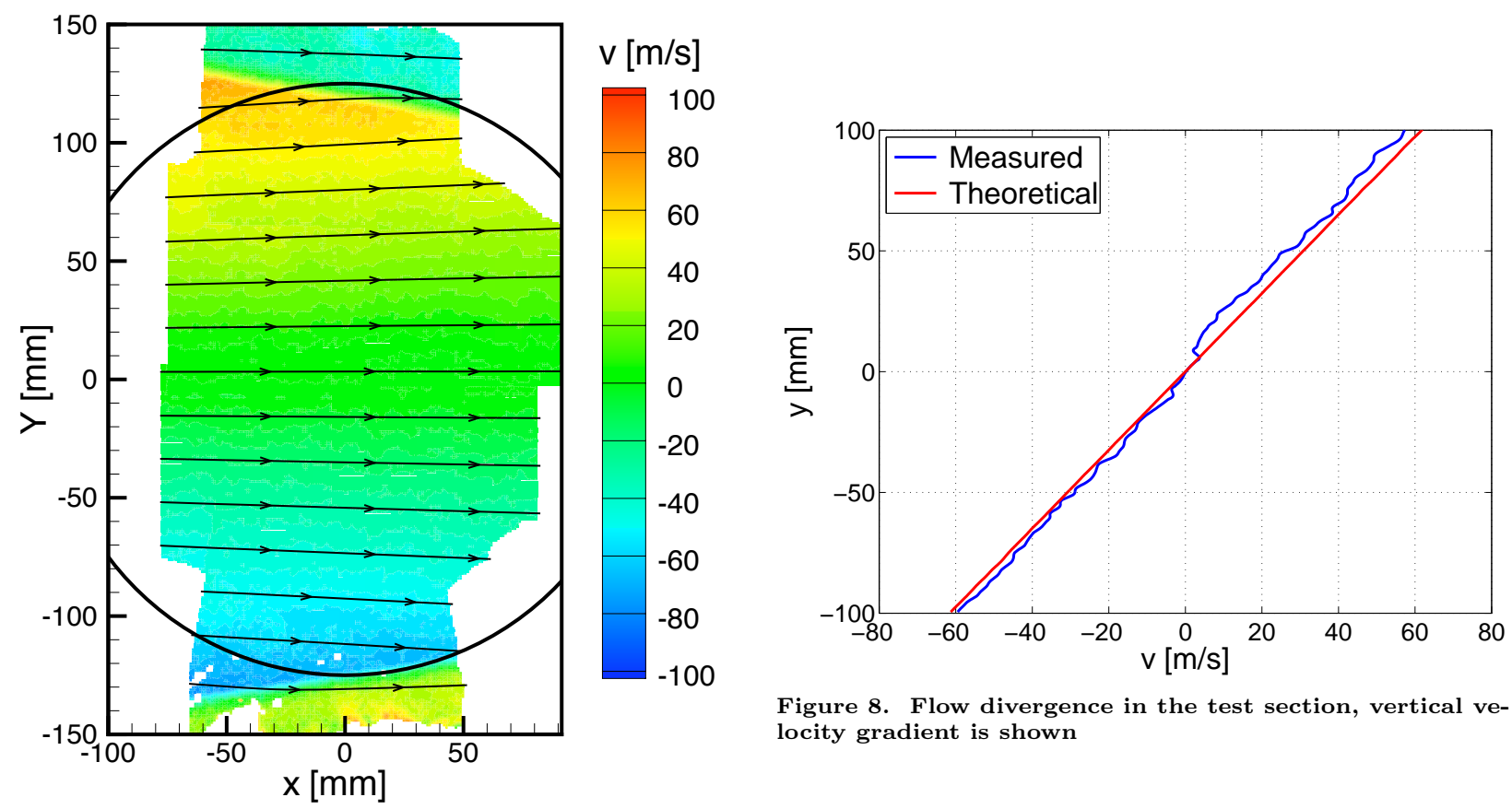

Figure 8. Flow divergence in the test section, vertical velocity gradient is shown

Figure 7. Vertical velocity component of the free stream flow for the Mach 7 nozzle

The flow divergence caused by the conical nozzle is also clearly measured. A profile of the vertical flow component in the center of the test section is plotted in figure 8. The vertical component varies from -60 to $+60 \mathrm{~m} / \mathrm{s}$ over a distance of $200 \mathrm{~mm}$. This complies well with the theoretical divergence corresponding to a total opening angle of $14^{\circ}$ indicated in figure 8 . 
A profile of the average total velocity magnitude in the center of the test section is given in figure 9 . The mean velocity is $1033 \mathrm{~m} / \mathrm{s}$ and the data show a uniformity of $0.2 \%$ (rms fluctuations within a single velocity field) and a repeatability of $0.4 \%$ (rms fluctuation with different realizations). In figure 10 the free stream velocity at the center line of the test section is given, the increase in velocity with downstream distance is caused by the diverging flow.

\section{IV.A. Temperature determination}

Since the free stream velocity and Mach number are known it is possible to deduce the static and total temperature. In hypersonic short duration facilities these two quantities are notoriously difficult to determine. However from the definition of the Mach number the static temperature can be directly determined:

$$
T=\frac{\left(\frac{|V|}{M}\right)^{2}}{\gamma R}
$$

The total temperature can now be calculated from the energy equation:

$$
T_{t}=T+\frac{|V|^{2}}{2 c_{p}}
$$

The resulting static and total temperature and given in table 5. For the determination of the temperatures the average velocity in the center of the test section is taken.

\begin{tabular}{|l|ll|}
\hline Free stream velocity & $\vec{V}=1033 \mathrm{~m} / \mathrm{s}$ \\
Free static temperature & $T=47 \mathrm{~K}$ \\
Free total temperature & $T_{t}=$ & $579 \mathrm{~K}$ \\
Free total enthalpy & $H_{0}=$ & $0.58 \mathrm{MJ}$ \\
\hline
\end{tabular}

Table 5. Free stream temperature for the Mach 7 nozzle

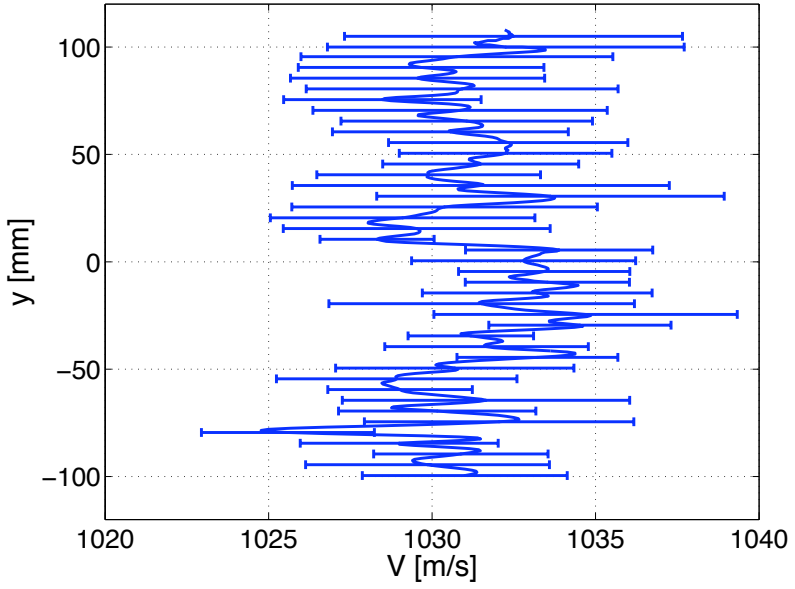

Figure 9. Vertical profile at $x=0$ of the free stream velocity for the Mach 7 nozzle

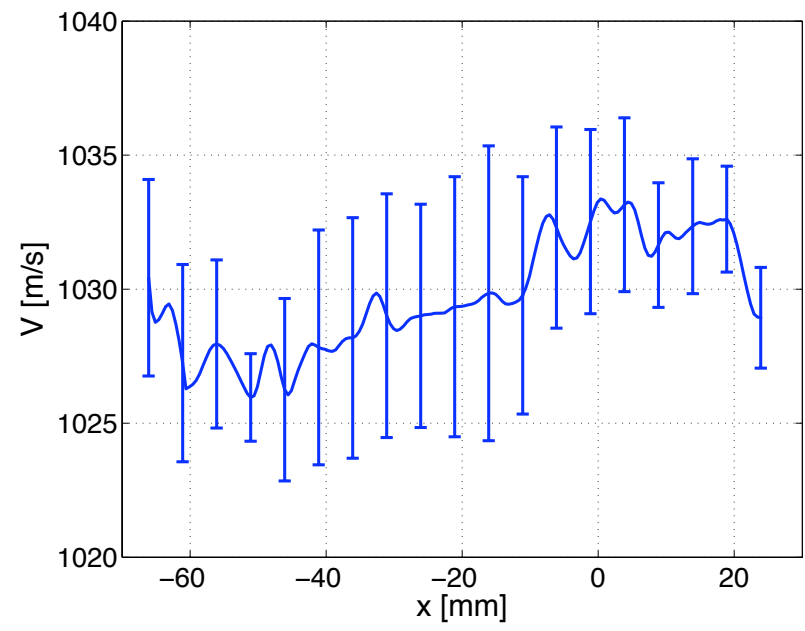

Figure 10. Horizontal profile at $y=0$ of the free stream velocity for the Mach 7 nozzle

It appears there is a large discrepancy between the measured total temperature and the storage temperature. In figure 11 the measured free stream velocity is given in blue as a function of the storage tube temperature, in red the corresponding free stream velocity is given for a fully adiabatic flow. There is a $10 \%$ difference between the measured and theoretical velocity. It is conceived that losses because of heat transfer in the valve and tandem nozzle are responsible for this.

\section{IV.B. Pressure measurements}

The free stream Mach number and total pressure is evaluated by means of pressure measurements. Static pressure measurements in the nozzle are performed in combination with Pitot tube measurements in the test 


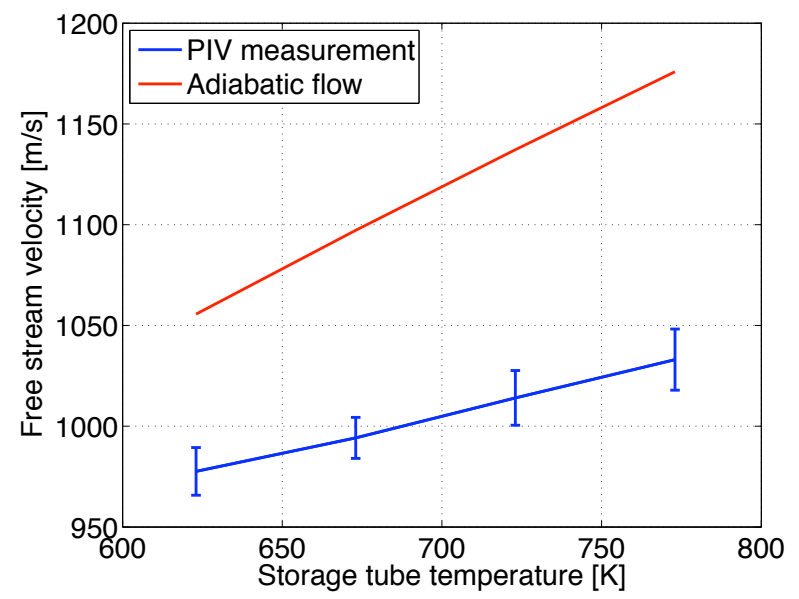

Figure 11. Variation of measured and theoretical free stream velocity with storage tube temperature

section. The Pitot tube formula correlates the measured Pitot pressure $p_{t, 2}$ to the total pressure $p_{t, 1}$ and the local Mach number $M_{r}$ (at the position of the Pitot tube):

$$
\frac{p_{t, 2}}{p_{t, 1}}=\left[\frac{\gamma+1}{2 \gamma M_{r}^{2}-(\gamma-1)}\right]^{\frac{1}{\gamma-1}}\left[\frac{(\gamma+1) M_{r}^{2}}{(\gamma-1) M_{r}^{2}+2}\right]^{\frac{\gamma}{\gamma-1}}
$$

The static pressure measurements also correlate the total pressure (which is considered to be constant throughout the nozzle and test section) and the local Mach number $M_{s}$ :

$$
\frac{p_{t, 1}}{p}=\left(1+\frac{\gamma-1}{2} M_{s}^{2}\right)^{\frac{\gamma}{\gamma-1}}
$$

To be able to solve the two equations it is assumed that the flow divergence is equal to the inviscid case corrected for the boundary layer displacement thickness. Now the local Mach number at the location of the static pressure orifices in the nozzle wall can be related to the Mach number at the Pitot tubes using the nozzle opening angle and the distance $\Delta x$ between the static pressure orifices and Pitot tubes. From geometry relations follows that:

$$
\frac{A_{s}}{A^{*}}=\left(\frac{r^{*} \sqrt{\frac{A_{r}}{A^{*}}}-\Delta x \tan \phi}{r^{*}}\right)^{2}
$$

where the local Mach number is related to the local cross section by the Mach area-ratio relation (see equation 10). Finally the equations are solved for $p_{t}$ and $M_{r}$.

In the measurements, 3 static pressure transducers were mounted at $[95,295,495] \mathrm{mm}$ from the nozzle test section junction, see figure 5 . The Pitot probe installed in the test section, is located $300 \mathrm{~mm}$ downstream of the junction. In the current procedure three static pressure signals are used in combination with two Pitot pressure signals in the optimization for finding $p_{t}$ and $M_{r}$. For the pressure signals given in figure 12 the following average values are obtained: $M_{r}=7.5 \pm 0.1$ and $p_{t}=27.9 \pm 1 \mathrm{bar}$, see also table 6

\begin{tabular}{|ccccc|cc|}
\hline$p_{s, 1}$ & $p_{s, 2}$ & $p_{s, 3}$ & $p_{r, 1}$ & $p_{r, 1}$ & $M$ & $p_{t}$ \\
\hline 0.01 & 0.0156 & 0.0273 & 0.3123 & 0.3197 & 7.5 & 27.9 \\
\hline
\end{tabular}

Table 6. Average measured pressure with the resulting Mach number and total pressure

\section{IV.C. Stagnation heatflux measurements}

In order to determine the free stream temperature for the nozzle setup without the tandem nozzle configuration (M9 nozzle), the stagnation heat flux of a $10 \mathrm{~cm}$ sphere-cylinder is measured by means of infrared 


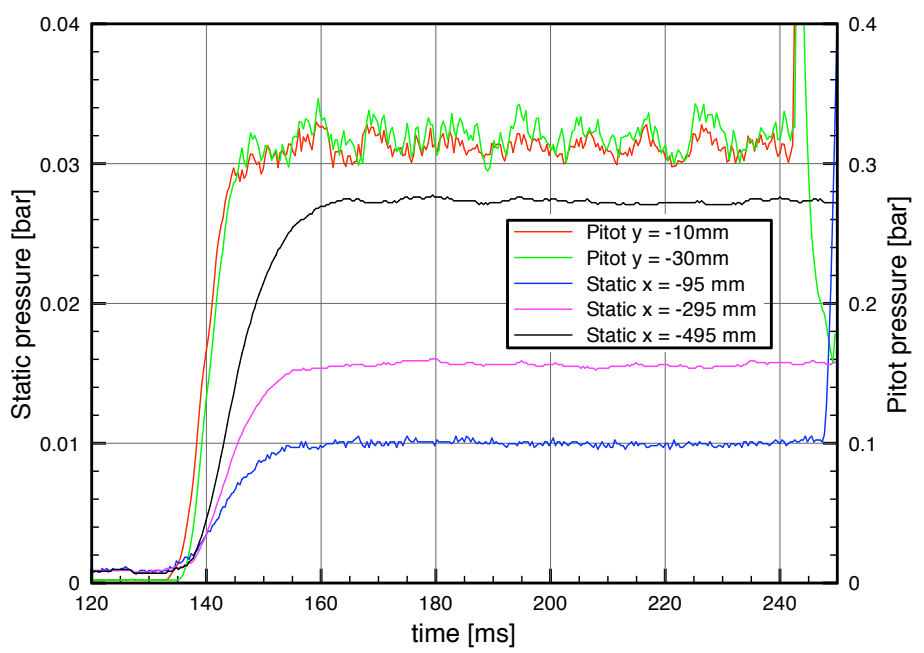

Figure 12. Static and Pitot pressure variation in time

thermography. Due to the presence of seeding particles in the flow, condensation occurred which eliminated the possibility to determine the static and total temperature by means of PIV. In figure 13 the heat flux variation in time is shown for different Reynolds numbers (total pressures). The horizontal lines in the figure

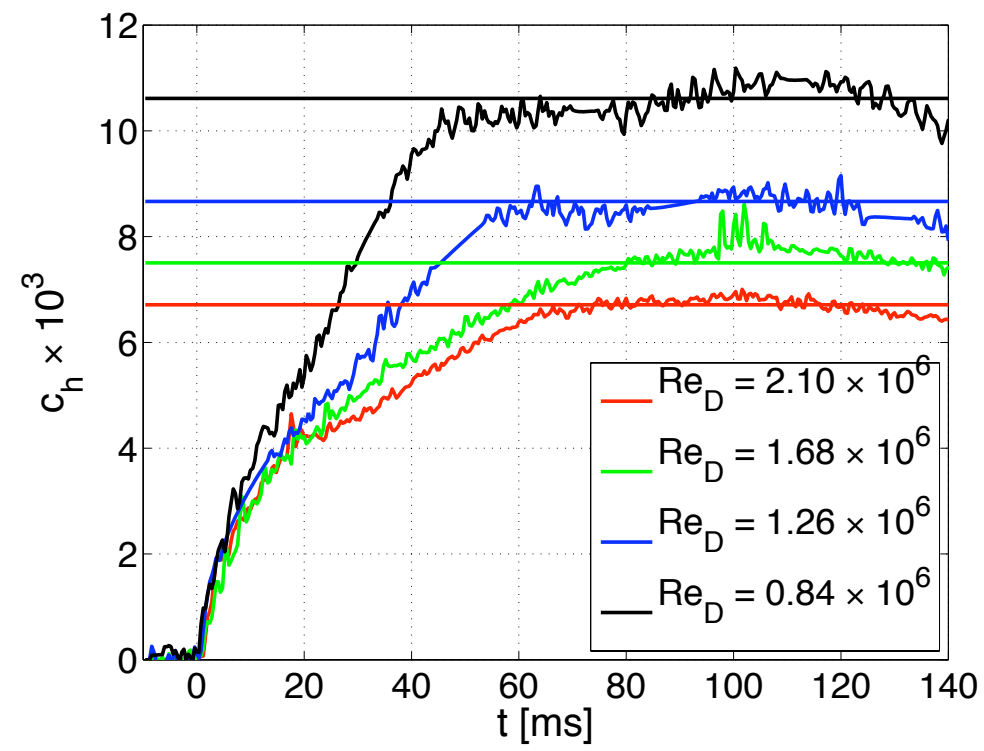

Figure 13. Measured stagnation point heat transfer compared to Fay-Riddell correlation results

are the theoretical values obtained using the Fay-Riddell stagnation point heat transfer correlation: ${ }^{12}$

$$
q=0.94\left(\rho_{e} \mu_{e}\right)^{0.4}\left(\rho_{w} \mu_{w}\right)^{0.1} \sqrt{\frac{\mathrm{d} u}{\mathrm{~d} x}}\left(h_{a w}-h_{w}\right)
$$

where the subscripts $e$ and $w$ denote the conditions at the boundary layer edge and the wall, respectively. The temperature difference used for the computation of $c_{h}$ in figure 13 is $T_{t}-T_{w}$ since the adiabatic wall temperature is difficult to obtain. The total temperature was varied to fit the Fay-Riddell results to the measured heat flux. A good agreement was found for $T_{t}=585 \mathrm{~K}$. The total temperature measured is higher than for the M7 nozzle. This is because for the M9 nozzle no tandem nozzle is required thus reducing the amount of heat lost in the process. 


\section{IV.D. Operational envelope}

The wind tunnel was extensively assessed for the Mach 7 nozzle and at a storage tube pressure of 100 bar. The displacement effect of the boundary layer was measured and the effect on the free stream Mach number was evaluated. Simultaneously the Mach number was obtained from static and Pitot tube measurements (section III). Both evaluations showed the test section Mach number to be $M=7.5$. The total pressure was inferred from the static-Pitot pressure measurements and the pressure measured just upstream of the second throat, the result was a total pressure of $p_{t}=27.9$ bar (section III). This also provided information on the efficiency of the tandem nozzle system regarding the total pressure loss. The free stream velocity was used to calculate the free stream total temperature $T_{t}=579 \mathrm{~K}$. Finally a free stream total temperature of $T_{t}=585 \mathrm{~K}$ was obtained for the Mach 9 nozzle by means of Fay-Ridell evaluations. An overview of the methods used to determine the free stream variables is shown in table 7 .

\begin{tabular}{|l|l|c|}
\hline Variable & Measurement method & Value \\
\hline \multirow{2}{*}{ Mach number $M$} & Theory with BL correction & $7.5 \pm 0.1$ \\
& Static-Pitot pressure measurement & $7.5 \pm 0.1$ \\
\hline \multirow{2}{*}{ Total pressure $p_{t}[$ bar $]$} & Pressure upstream of the throat & $28.6 \pm 1$ \\
& Static-Pitot pressure measurement & $27.9 \pm 1$ \\
\hline \multirow{2}{*}{ Total temperature $T_{t}[K]$} & Velocity by means of PIV in M7 nozzle & 579 \\
& Fay-Ridell evaluation in M9 nozzle & 585 \\
\hline
\end{tabular}

Table 7. Overview of methods to assess the free stream characteristics of the HTFD

The free stream characteristics for the Mach 7 nozzle are used to establish the Mach-Reynolds operational envelope of the HTFD. The free stream Mach number is obtained from the theoretical area ratio corrected for the displacement effect of the nozzle boundary layer. In Lukasiewicz ${ }^{13}$ an empirical correlation is given for the variation of displacement thickness with the Reynolds number based on the reference temperature:

$$
\frac{\delta^{*}}{x}=0.42 R e_{\text {ref }}^{-0.2775}
$$

This correlation is used in combination with the conditions the Mach 7 nozzle to obtain the displacement thickness at other Mach numbers and total pressures:

$$
\frac{\delta^{*}}{\left(\delta^{*}\right)_{M 7}}=\left\{\frac{R e_{\mathrm{ref}}}{\left(R e_{\mathrm{ref}}\right)_{M 7}}\right\}^{-0.2775}
$$

The maximum total pressure is determined by the maximum allowable pressure in the storage tube and the losses when the tandem nozzle is installed. The minimum pressure is determined by the pressure ratio needed to operate the tunnel at the given Mach number. The total temperature is $T_{t}=585 K$ without the tandem nozzle and $T_{t}=579 \mathrm{~K}$ with the tandem nozzle installed.

\begin{tabular}{|c|cccc|}
\hline Nozzle & $M$ & $p_{t}[\mathrm{bar}]$ & $T_{t}[\mathrm{~K}]$ & $R e / m \times 10^{6}\left[\mathrm{~m}^{-1}\right]$ \\
\hline \multirow{2}{*}{ M6 } & 6.4 & 2.8 & 579 & 7.90 \\
& 6.5 & 14.3 & 579 & 1.61 \\
\hline \multirow{2}{*}{ M7 } & 7.4 & 5.4 & 579 & 11.05 \\
& 7.5 & 28.0 & 579 & 2.22 \\
\hline \multirow{2}{*}{ M8 } & 8.4 & 10.0 & 579 & 15.08 \\
& 8.5 & 51.2 & 579 & 3.07 \\
\hline \multirow{2}{*}{ M9 } & 9.4 & 20 & 585 & 19.70 \\
& 9.5 & 88 & 585 & 4.65 \\
\hline \multirow{2}{*}{ M10 } & 10.3 & 20 & 585 & 15.85 \\
& 10.5 & 88 & 585 & 3.76 \\
\hline
\end{tabular}

Table 8. Free stream total quantities for different nozzles and total pressures 
The free stream total quantities as well as Mach and unit Reynolds number for different nozzle geometries is given in table 8 and in figure 14. The total temperature is fixed at the maximum allowed for condensation issues, however for the lower Mach numbers (M6 and M7 nozzles) the temperature could be reduced to achieve a higher Reynolds number.

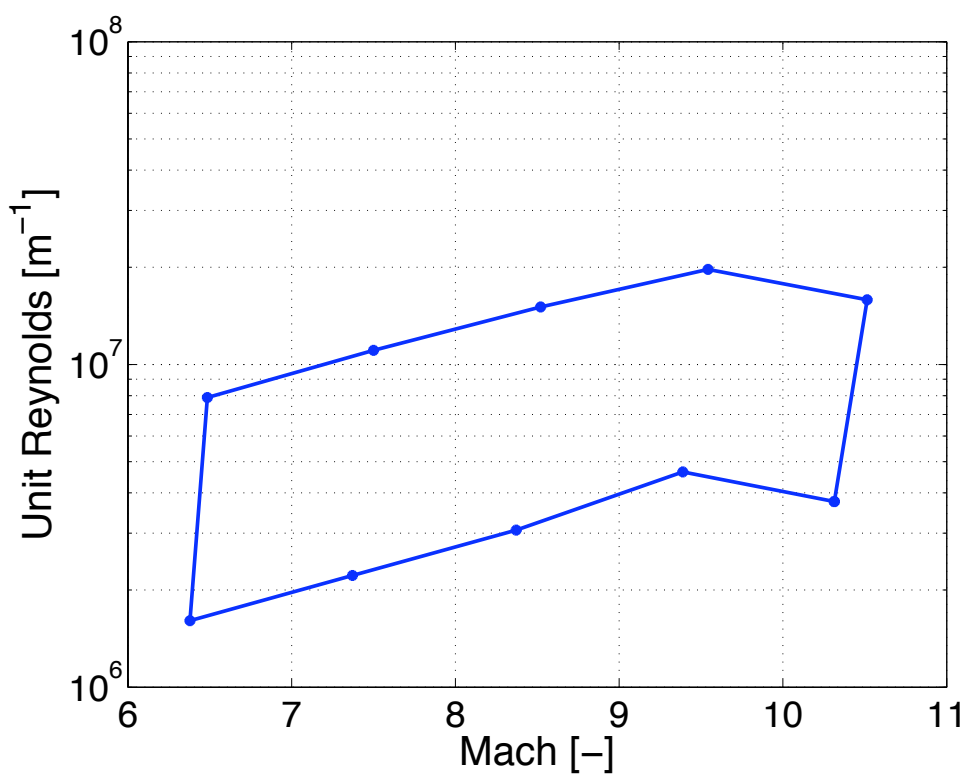

Figure 14. Operational Mach Reynolds envelope

\section{IV.E. Condensation}

Since the free stream static temperature is relatively low the free stream flow should be checked for condensation effects. In general two types of condensation processes are possible: heterogeneous condensation that behaves like normal equilibrium condensation and spontaneous condensation for which supercooling is possible. Heterogeneous condensation is characterized by the process where the presence of $\mathrm{CO}_{2}$ and $\mathrm{H}_{2} \mathrm{O}$ content in air is sufficient for causing condensation of nitrogen and oxygen through a seeding action. Spontaneous condensation is characterized by the nucleation of the nitrogen component which then acts as nucleus for the rest of the nitrogen and oxygen. The spontaneous condensation process is governed by the ratio of expansion rate to static pressure, $\frac{\dot{P}}{p},{ }^{14}$ which judges the amount of supercooling. For the free stream it is given by:

$$
\frac{\dot{P}}{p}=\frac{\sqrt{\gamma R T_{t}}}{p_{t}} \frac{2 \gamma M^{4}}{M^{2}-1} \frac{r \tan \phi}{r^{* 2}}
$$

where $\dot{p}=-(1 / p)(d p / d t), r$ is the local radius and $r^{*}$ is the throat radius. In case $\frac{\dot{P}}{p}$ is smaller than about $0.1-1 s^{-1} \mathrm{mbar}^{-1}$ little or no supercooling is expected since the process is dominated by heterogeneous condensation. For HTFD the value of $\frac{\dot{P}}{p}$ is around $3-50 \mathrm{~s}^{-1} \mathrm{mbar}^{-1}$ where the high values are obtained for the higher Mach numbers. From this it can be concluded that the condensation process is dominated by spontaneous condensation and supersaturated conditions can be achieved.

In figure 15 a pressure-temperature diagram is given in which data is depicted for condensation onset in hypersonic facilities. ${ }^{14}$ In the figure also the mean onset of nitrogen as obtained from experiments is depicted. In general the expansion rate decreases with increasing nozzle size therefore the amount of supercooling for large facilities is reduced resulting in an earlier condensation onset. ${ }^{13}$ For low static pressures $(\approx 1 \mathrm{mbar})$ the seeding effect of $\mathrm{CO}_{2}$ and $\mathrm{H}_{2} \mathrm{O}$ is negligible and spontaneous condensation dominates the process. The black dots indicate the HTFD static conditions at different Mach numbers. For the M7 nozzle the static conditions are still within the equilibrium condensation limits for $N_{2}$. At higher Mach numbers static temperatures are achieved for which equilibrium condensation would occur. From our experience it may be concluded that a free stream without condensation may be achieved for the M9 nozzle. 


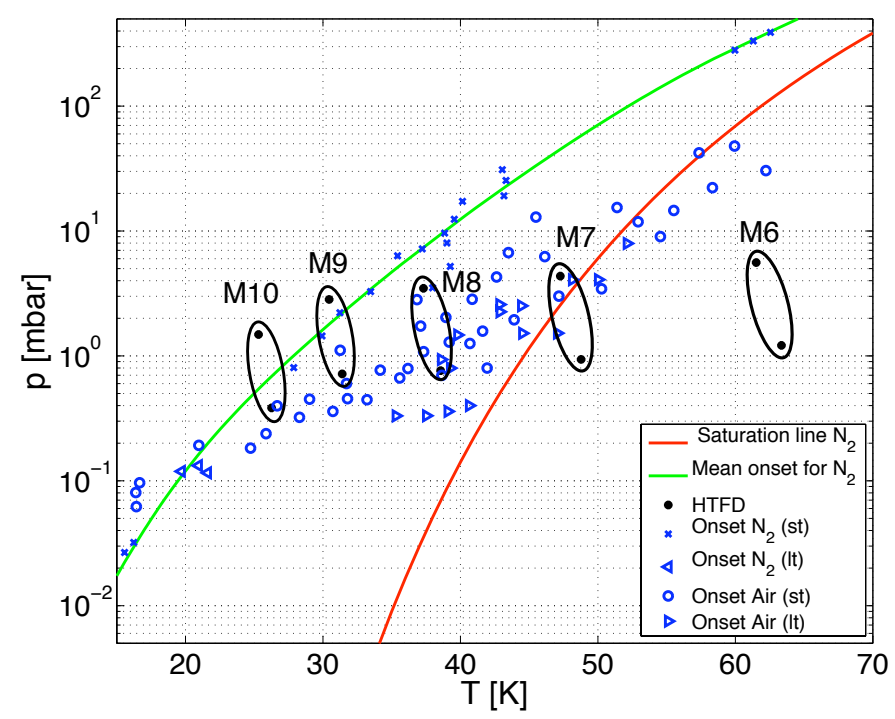

Figure 15. Experimental estimates of condensation onset ${ }^{13,14}$ ('st' means small tunnel $<30 \mathrm{~cm}$ and 'lt' means large tunnel $>130 \mathrm{~cm}$ )

\section{Conclusion}

The lay-out and working of the Hypersonic Test Facility Delft (HTFD) has been given. The performance of the facility was verified against theory by means of pressure measurements, velocity measurements (Particle Image Velocimetry, PIV) and infrared thermography.

The free stream Mach number, total pressure and total temperature were all determined in two independent ways. The nozzle boundary layer displacement thickness was measured and used to correct the geometrical cross section for the determination of the free stream Mach number. Additionally it was also inferred from static-Pitot pressure measurements. These measurements also allowed to obtain the total free stream pressure. It was found that this matched the total pressure measured in the tandem nozzle. The total and static temperature was obtained from PIV velocity measurements for the M7 nozzle. In case of the M9 nozzle the temperature was obtained using Fay-Ridell evaluation.

It was shown that the experimental achievements as obtained by the mechanical quantities pressure and velocity met the predicted theoretical expectations. However, the temperature did not come off as favorable. The total temperatures measured in the storage tube differed considerably from the prediction obtained from the PIV velocity measurements and the Mach numbers using the Mach area ratio relation. This deficiency may be attributed to the large unknown heat transfer at the passage of the flow from storage tube via the complex mechanism containing the fast acting valve to the nozzle. This aspect is possibly considered unsatisfactorily in the literature of similar facilities as the present one.

\section{Acknowledgments}

The authors would like to acknowledge F.Scarano for his advice and they also thank R.Caljouw for his help in performing the experiments. This work is supported by the Dutch Technology Foundation STW under the VIDI Innovation Impulse program, grant DLR.6198.

\section{References}

\footnotetext{
${ }^{1}$ Ludwieg, H., "Der Rohrwindkanal," Zeitschrift für Flugwissenschaften, Vol. 3, 1955, pp. 206 - 216. 1968.

${ }^{2}$ Hottner, T., "Der Rohrwindkanal der Aerodynamischen Versuchsanstalt Göttingen," Tech. rep., AVA 68A 77, Göttingen,

${ }^{3}$ Hottner, T., "Eichmessungen im Rohrwindkanal $500 \varnothing$ der AVA," Tech. rep., AVA 69A23, Göttingen, 1969.

${ }^{4}$ Wolf, T., Estorf, M., and Radespiel, R., "Investigation of the starting process in a Ludwieg tube," Theor. Comput. Fluid Dyn., Vol. 21, 2007, pp. 81-98.
} 
${ }^{5}$ Liepmann, H. and Roshko, A., Elements of gasdynamics, Dover publications inc., 2001.

${ }^{6}$ Staff, A. R., "Equations, tables, and charts for compressible flow," report 1135, NACA, 1953.

${ }^{7}$ Friehmelt, H., Koppenwallner, G., and Muller-Eigner, R., "Calibration and first results of a redesigned Ludwieg expansion tube," AIAA-paper 93-5001, AIAA, 1993.

${ }^{8}$ Scarano, F. and van Oudheusden, B., "Planar velocity measurements of a two-dimensional compressible wake," Experiments in Fluids, Vol. 34, 2006, pp. 430-441.

${ }^{9}$ Schrijer, F., Scarano, F., and van Oudheusden, B., "Application of PIV in a Mach 7 double-ramp flow," Experiments in Fluids, Vol. 41, 2006, pp. $353-363$.

${ }^{10}$ Prasad, A., "Stereoscopic particle image velocimetry," Experiments in Fluids, Vol. 29, 2000, pp. 103-116.

${ }^{11}$ White, F. M., Viscous fluid flow, McGraw-Hill, Singapore, 2nd ed., 1991.

12 Anderson, Jr., J. D., Hypersonic and high temperature gas dynamics, McGraw-Hill, Boston, Massachusetts, 1989.

${ }^{13}$ Lukasiewicz, J., Experimental methods of hypersonics, Vol. 3 of Gasdynamics, Marcel Dekker Inc, New York, 1973.

${ }^{14}$ Daum, F. and Gyarmathy, G., "Condensation of air and nitrogen in hypersonic wind tunnels," AIAA journal, Vol. 6, No. 3, 1968, pp. $458-465$. 